\title{
Unlocking High-Salinity Desalination with Cascading Osmotically Mediated Reverse Osmosis: Energy and Operating Pressure Analysis
}

\author{
Xi Chen ${ }^{\dagger}$ and Ngai Yin Yip*, ${ }^{\dagger}+\infty$ \\ ${ }^{\dagger}$ Department of Earth and Environmental Engineering, Columbia University, New York, New York 10027-6623, United States \\ "Columbia Water Center, Columbia University, New York, New York 10027-6623, United States
}

Supporting Information

ABSTRACT: Current practice of using thermally driven methods to treat hypersaline brines is highly energy-intensive and costly. While conventional reverse osmosis (RO) is the most efficient desalination technique, it is confined to purifying seawater and lower salinity sources. Hydraulic pressure restrictions and elevated energy demand render RO unsuitable for high-salinity streams. Here, we propose an innovative cascading osmotically mediated reverse osmosis (COMRO) technology to overcome the limitations of conventional RO. The innovation utilizes the novel design of bilateral countercurrent reverse osmosis stages to depress the hydraulic pressure needed by lessening the osmotic pressure difference across the membrane, and simultaneously achieve energy savings. Instead of the 137 bar required by conventional RO to desalinate $70000 \mathrm{ppm}$ TDS hypersaline feed, the highest operating pressure in COMRO is only 68.3

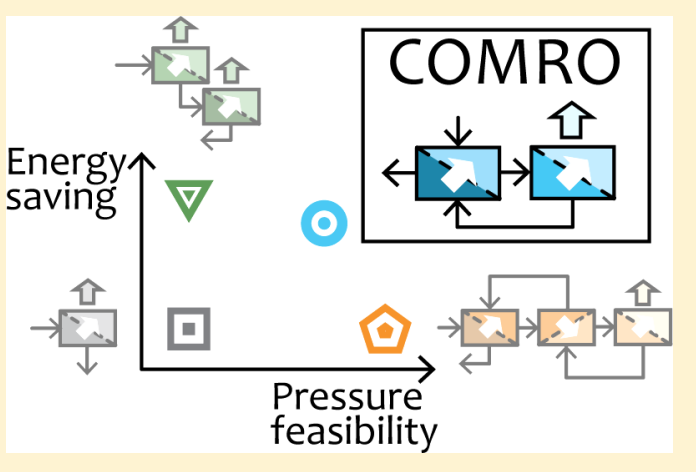
bar $(-50 \%)$. Furthermore, up to $\approx 17 \%$ energy saving is attained by COMRO $\left(3.16 \mathrm{kWh} / \mathrm{m}^{3}\right.$, compared to $3.79 \mathrm{kWh} / \mathrm{m}^{3}$ with conventional RO). When COMRO is employed to boost the recovery of seawater desalination to $70 \%$ from the typical $35-50 \%$, energy savings of up to $\approx 33 \%$ is achieved $\left(2.11 \mathrm{kWh} / \mathrm{m}^{3}\right.$, compared to $3.16 \mathrm{kWh} / \mathrm{m}^{3}$ with conventional RO). Again, COMRO can operate at a moderate hydraulic pressure of $80 \mathrm{bar}$ ( $25 \%$ lower than $113 \mathrm{bar}$ of conventional RO). This study highlights the encouraging potential of energy-efficient COMRO to access unprecedented high recovery rates and treat hypersaline brines at moderate hydraulic pressures, thus extending the capabilities of membrane-based technologies for high-salinity desalination.

\section{INTRODUCTION}

Management and treatment of high-salinity brines, such as produced water from oil and gas industries and waste streams of minimum/zero liquid discharge (MLD/ZLD) operations, have rapidly risen to be major environmental challenges. ${ }^{1-6}$ Underground injection of produced water, the predominant practice, ${ }^{7}$ is expensive, environmentally unsustainable, and not always available. ${ }^{1,3,8,9}$ At the same time, rising costs and impending regulations on wastewater and brine disposal, together with mounting strain on freshwater resources provide strong impetuses for the development of MLD/ZLD technologies. ${ }^{4,5,10}$

Very high total dissolved solids (TDS) concentrations (> $\approx 70000 \mathrm{ppm}$ ) pose considerable technical challenges in treatment. Currently, thermal separation methods of mechan$\mathrm{ical} /$ thermal vapor compression, multiple effect distillation, thermal brine concentrator, and evaporation pond are the prevailing techniques to desalinate or dewater highly concentrated brines. ${ }^{1,6,11}$ However, these thermally driven processes are inherently inefficient and highly energy intensive. ${ }^{12-14}$ Membrane-based technologies, including membrane distillation (MD) and forward osmosis (FO), have been advanced as alternatives. ${ }^{1,5,15-19}$ But MD is ultimately still a heat-driven distillation process, whereas FO draw agents used in pilot demonstrations are also thermally regenerated. ${ }^{15,16}$
Therefore, the overall energy efficiency of both MD and FO are still constrained by unfavorable thermodynamic limitations governing all thermal separations. ${ }^{20-22}$

Reverse osmosis (RO), an isothermal separation, is the most energy-efficient method for seawater and brackish water desalination. ${ }^{23-26}$ But, RO typically handles streams up to only $\approx 70000$ ppm TDS (effluent salinity), approximately $2 \times$ seawater concentration. Greater salinities require higher hydraulic pressures to overcome the osmotic pressure that scales with TDS. However, studies have reported severe deterioration of membrane transport properties under high pressurizations. $^{27-30}$ Furthermore, because the energy consumption of conventional single-stage RO increases with feed salinity and recovery yield, treating hypersaline brines, and desalinating to high recovery rates (i.e., produced water and MLD/ZLD applications, respectively) will be substantially more energy intensive. ${ }^{26}$

Alternative system configurations of serially staged RO and closed-circuit RO have been proposed to reduce desalination energy. ${ }^{31,32}$ While analyses indicate that these configurations

Received: November 10, 2017

Revised: January 12, 2018

Accepted: January 22, 2018

Published: January 22, 2018 
can provide energy savings, both still need high hydraulic pressures that are not typically utilized in conventional RO operation. The development of new membrane-based technologies capable of treating high-salinity feed streams with moderate hydraulic pressure requirements will have important impacts on desalination. Such innovations will supplant costly thermal processes for treating hypersaline streams of, for example, produced water and MLD/ZLD brine.

In this study, we propose a novel cascading osmotically mediated reverse osmosis (COMRO) technology to overcome the limitations of conventional RO and enable high-salinity desalination. The technique utilizes the innovative design of bilateral countercurrent reverse osmosis stages to substantially lower the hydraulic pressure needed for operation and simultaneously achieve energy reductions. The working principles of COMRO first are introduced. Analytical expressions for the minimum specific energy consumption and hydraulic pressure requirement are derived for COMRO and alternative RO configurations. The study then quantifies the energy savings and reduced hydraulic pressure benefits of COMRO for two scenarios of hypersaline brine desalination and high recovery seawater desalination. Capital cost considerations on levelized cost of water production are discussed and an example of rapid COMRO implementation is presented.

\section{CASCADING OSMOTICALLY MEDIATED REVERSE OSMOSIS}

Practical Limitations of Conventional Reverse Osmosis. While RO has emerged as the most efficient seawater desalination technology, ${ }^{20,24-26}$ there are practical constraints limiting the range of $\mathrm{RO}$ operation. Figure $1 \mathrm{~A}$ shows a schematic of a conventional single-stage RO, where a highpressure pump drives water permeation across the membrane module, yielding freshwater as product. Energy embedded in the pressurized exiting brine stream is captured with an energy recovery device (ERD), e.g., pressure exchanger. The constant hydraulic pressure required for operation, $\Delta P$, must be greater than the osmotic pressure of the brine effluent, which scales with the TDS concentration.

High hydraulic pressures have been reported to have detrimental and oftentimes irreversible impacts on membrane transport properties, including water permeability loss and compromised selectivity. ${ }^{27-30}$ Thus, RO is traditionally restricted to pressures $<85$ bar $(\approx 1200 \mathrm{psi}),{ }^{33,34}$ which imposes upper bounds on the brine effluent concentration and, in turn, the recovery rate $Y$ (defined as the ratio of the product water flow rate to feedwater flow rate). ${ }^{33}$ Because of the vulnerability of membranes to diminished performance at high pressurizations, conventional $\mathrm{RO}$ is, therefore, unsuitable for the desalination of high-salinity feed streams. For seawater desalination, $\Delta P$ limitation is one of the factors restricting typical recovery rates to $35-50 \%{ }^{23,24,35}$

Even if the hydraulic pressure limitation can be overcome with advances in membrane material and module design to substantially improve mechanical robustness, it will be challenging to feasibly employ RO for the desalination of hypersaline feed or to achieve higher recoveries. This is because $\mathrm{RO}$ energy requirement is directly proportional to $\Delta P^{26,36}$ Operating conventional single-stage RO at ultrahigh hydraulic pressures will, thus, be penalized by excessively large energy cost, severely weakening the economic favorability.
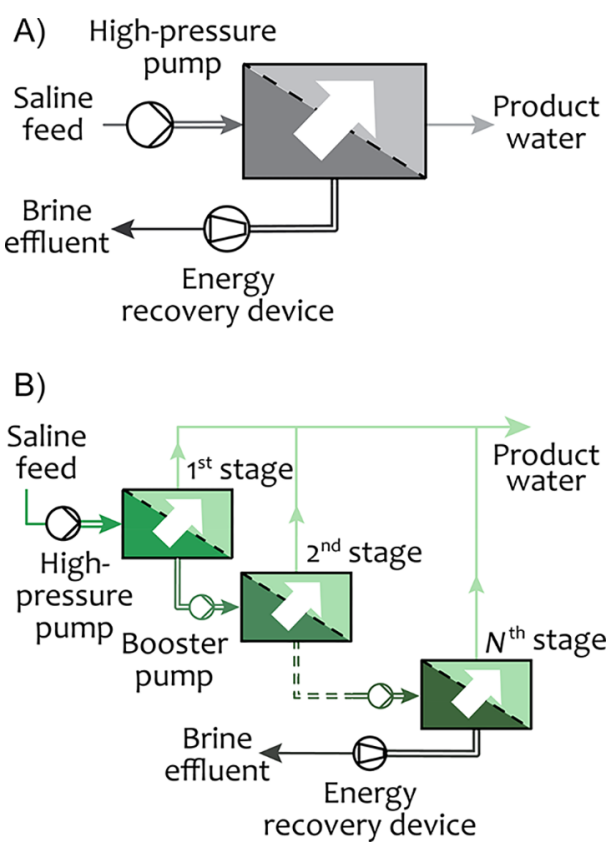

Figure 1. Schematics of (A) conventional single-stage RO and (B) direct pass RO (DPRO) with $N$ stages, where the retentate stream of each stage is further pressurized by a booster pump and directed to the next stage as influent. The stepped increase in hydraulic pressure along the series drives water permeation in each stage, cumulatively yielding product water and concentrating the retentate stream into the brine effluent for discharge. Color intensity of the streams and stages is representative of the salinity, while the white block arrows in the membrane modules indicate direction of water permeation. Double and single lines denote pressurized streams and ambient pressure streams, respectively.

Working Principles of DPRO. With current state-of-the-art single-stage RO approaching the thermodynamic limit in energy efficiency for seawater desalination, ${ }^{26,37}$ alternative system designs are being considered for reducing energy consumption. Analytical studies indicate that multistage direct pass reverse osmosis (DPRO) is more energy efficient than conventional single-stage RO. ${ }^{26,32,38}$ In DPRO, $N$ repeating RO stages are connected in series (Figure 1B). The retentate stream of one stage is further pressurized by a booster pump and directed to the next stage as feed. Water permeation across the membrane in each stage cumulatively yields the product water and gradually concentrates the retentate stream into brine effluent that is eventually post-treated and discharged.

Progressing along the stages in DPRO, the retentate stream osmotic pressure of each stage increases stepwise, raising the required $\Delta P$ correspondingly. The osmotic pressure before the last stage will, hence, always be lower than that of the final brine effluent. Therefore, $\Delta P$ in all stages, except the final stage, is lower than the applied hydraulic pressure in a single-stage RO with the same overall $Y$. As the average applied pressure weighted by the permeate volume of each stage in DPRO is reduced compared to single-stage RO operation, desalinating with a series of stages can be more efficient than conventional single-stage RO. Nevertheless, in DPRO the highest hydraulic pressure required, $\Delta P_{\max }$ is still dictated by the osmotic pressure of final brine effluent and, hence, remains the same as single-stage RO. Therefore, the constraints imposed by high hydraulic pressures, discussed above, remain unresolved. 


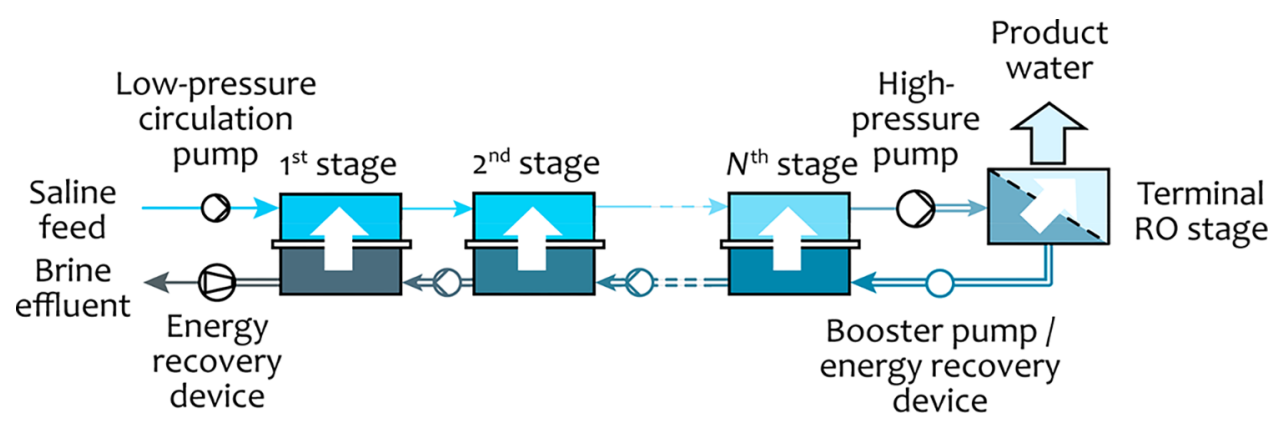

Figure 2. Schematic diagram of COMRO with $N$ units of BCC stages. The input feed is diluted in a series of bilateral countercurrent (BCC) reverse osmosis stages before passing through a terminal standard RO module to produce freshwater using only moderate hydraulic pressures. The retentate stream is cycled back to the cascading BCC stages in countercurrent flow, and the applied hydraulic pressure of each stage drives water permeation across the membrane, concentrating the retentate stream to a hypersaline brine for discharge. Color intensity of the streams and stages is representative of the salinity, while the white block arrows in the membrane modules indicate direction of water permeation. Double and single lines denote pressurized streams and ambient pressure streams, respectively.

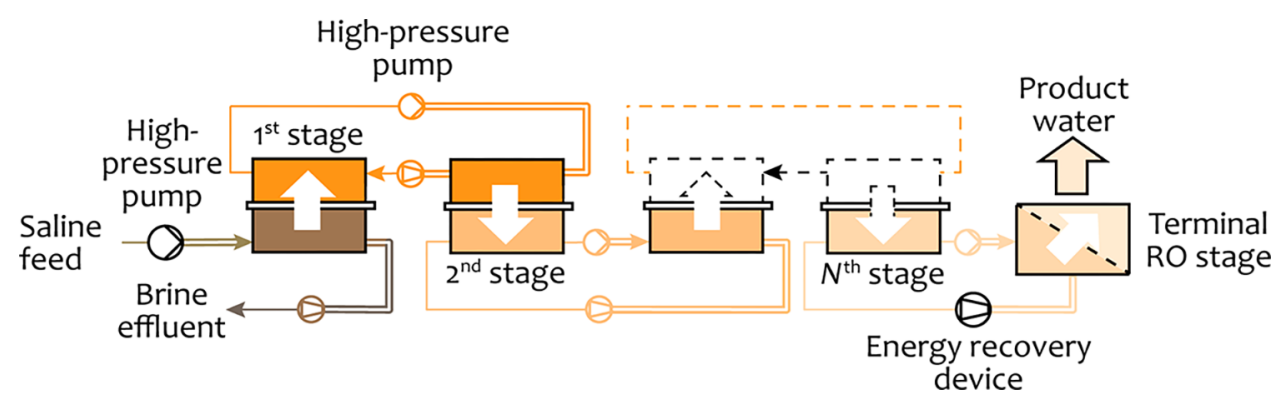

Figure 3. Schematic of CF/OARO with $N$ bilateral stages and terminated with a standard RO stage, connected by $N$ recirculating loops of working solution with progressively lower osmotic pressure. The streams within each loop are pressurized before being sent to the next stage and then fully depressurized before being recirculated back. Freshwater is produced in the terminal RO stage from a more dilute influent than the input feed stream, hence requiring a lower applied hydraulic pressure. Color intensity of the streams and stages is representative of the salinity, while the white block arrows in the membrane modules indicate direction of water permeation. Double and single lines denote pressurized streams and ambient pressure streams, respectively.

Working Principles of COMRO. To overcome the practical limitations of $\mathrm{RO}$ and extend the technology to desalinate higher salinity feeds and/or achieve greater recovery rates, we propose a novel cascading osmotically mediated reverse osmosis (COMRO). Working principles of COMRO are illustrated by the schematic in Figure 2. The unpressurized saline feed is introduced to the bilateral countercurrent (BCC) stages with a circulation pump. Unlike conventional RO with feed/retentate on one side and permeate on the other, the BCC stages have crossflow of saline streams on both sides in addition to the applied hydraulic pressure, $\Delta P$. The feed stream is sequentially diluted in the $N$ repeating BCC stages by water permeating from the opposite side. The diluted stream is then pressurized to moderate $\Delta P$ and desalinated in a terminal $\mathrm{RO}$ stage, i.e., standard conventional RO module, to yield fresh product water. The retentate stream exiting the terminal $\mathrm{RO}$ stage is cycled back to the cascading BCC stages in countercurrent flow, with booster pumps to incrementally ramp up the hydraulic pressure applied on the stream. Because the osmotic pressure difference across the membrane, $\Delta \pi$, is mediated by the dilute stream on the other side, $\Delta P$ needed to drive water permeation from the retentate stream (lower half of BCC stages), across the membrane to the upper half, is significantly lowered. The retentate stream is progressively concentrated along the cascade due to water flux in the BCC stages. The eventual hypersaline brine effluent generated is depressurized using an ERD prior to post-treatment and discharge.
The dilute stream in the BCC stages, i.e., upper half of the cascade, is at low hydraulic pressures (close to ambient) for circulation, and is pressurized by the high-pressure pump of the terminal RO stage. The retentate stream remains pressurized in the lower half of the cascading BCC stages, with $\Delta P$ increased stepwise by booster pumps (or, depending on operating condition, lowered by an ERD going into the $N^{\text {th }}$ stage). Because the salinity of the upper half stream osmotically mediates the hydraulic pressure needed in the BCC stages, the retentate stream can, hence, be concentrated to very high salinities without $\Delta P$ exceeding the operating pressure limit. Likewise, the dilution of the influent stream by water permeation in the cascade of BCC stages considerably lowers the hydraulic pressure needed for water production in the terminal RO stage. Therefore, feedwater of salinities substantially greater than seawater can be desalinated in COMRO, and recovery rates $>50 \%$ can be attained with seawater.

Working Principles of Counter Flow/Osmotically Assisted RO. Another design that utilizes dilute saline streams to mediate $\Delta \pi$ was recently proposed, and is termed "counter flow reverse osmosis (CFRO)"39,40 or "osmotically assisted reverse osmosis (OARO)" ${ }^{41}$ Working principles of CF/OARO are detailed elsewhere, ${ }^{40,41}$ and are briefly explained here. CF/ OARO comprises $N$ bilateral stages and a terminal RO stage connected by $N$ recirculating working solution loops (Figure 3). The pressurized saline feed is introduced to the first bilateral stage, whereas an unpressurized low concentration working solution (e.g., $\mathrm{NaCl}$ solution) is circulated on the opposite 


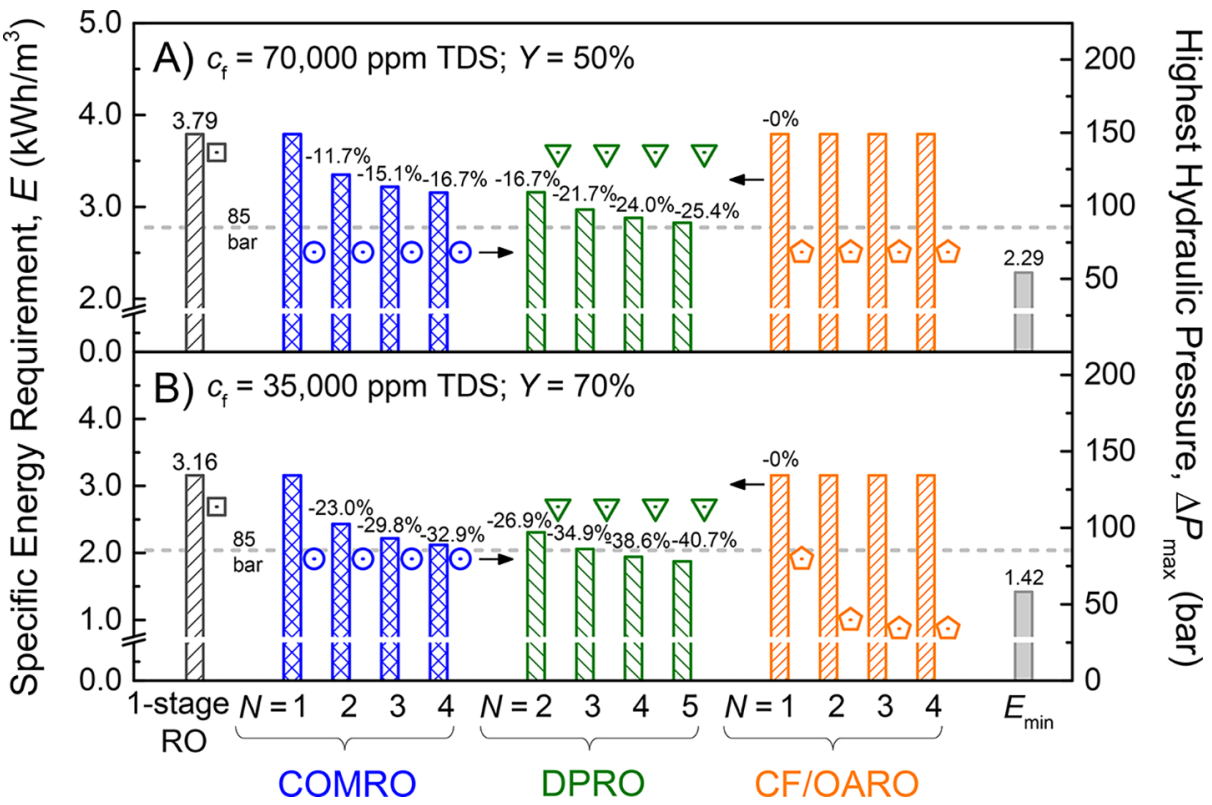

Figure 4. Specific energy requirement, $E$ (columns, left vertical axis), and highest hydraulic pressure, $\Delta P_{\max }$ (symbols, right vertical axis), of conventional single-stage RO, COMRO, DPRO, CF/OARO, and $E_{\min }$ for (A) desalination of hypersaline feed at $70000 \mathrm{ppm}$ TDS to $50 \%$ recovery and (B) desalinating seawater at 35,000 ppm TDS to high recovery of 70\%. Multistaged processes COMRO and CF/OARO were evaluated for $N=$ $1-4$, while DPRO with $2-5$ stages were assessed. Note that for the same total number of stages (including terminal RO stage), $N_{\mathrm{COMRO} / \mathrm{CF} / \mathrm{OARO}}+1$ $=N_{\text {DPRO }}$. Gray dotted lines denote 85 bar, the upper hydraulic pressure operating range of typical membrane modules. Osmotic pressures are determined using the van't Hoff equation with $\mathrm{NaCl}$ solutions at temperature of $298 \mathrm{~K}$, and the operational overpressurization factor, $k$, is 1.15 for all configurations. Pump and ERD inefficiencies, parasitic pressure drops along membrane modules, pre- and post-treatment costs, and intake and outfall power consumption are not factored into the energy requirement calculations.

permeate side as the sweep stream. The osmotically mediated $\Delta P$ drives water permeation across the membrane and the sweep stream is diluted, whereas the retentate is concentrated and eventually discharged as brine effluent. The diluted first sweep stream is then pressurized and cycled to the second bilateral stage as the feed influent. A second sweep stream of a more dilute working solution flows unpressurized in the permeate side of the second stage (i.e., similar to first stage operation). Water permeates across the second stage due to the net driving force of $\Delta P-\Delta \pi$. The first sweep stream, now concentrated, is then depressurized and recycled back to the first stage permeate side, whereas the diluted second sweep stream is pressurized and directed to the third stage.

These configurations of looping stages and pressurizationdepressurization operation of working solutions are repeated down the process train (Figure 3), with the sweep stream concentration gradually lowered along the stages and loops. The $N^{\text {th }}$ sweep stream, after successive dilution in the bilateral stages, is finally channeled to a terminal conventional RO stage to yield fresh product water. Like COMRO where $\Delta \pi$ is mediated by a more dilute stream, the $\Delta P$ needed to drive water permeation in the bilateral stages of $\mathrm{CF} / \mathrm{OARO}$ is similarly reduced. In order to achieve steady-state throughout the whole process, the permeation rate must be maintained precisely identical in all stages, including the terminal RO stage, such that there is no net accumulation or depletion of stream volume in any of the loops. Note that while the BCC stages in COMRO are configured in cascading countercurrent flow, the $\mathrm{CF} / \mathrm{OARO}$ stages are linked by closed recirculating loops. Another critical difference is that working solutions are employed in the closed loops to achieve the necessary stepwise dilution in CF/OARO, but in COMRO the diluted influent feed is cycled back down the stages to be the retentate stream (Figures 2 and 3).

\section{ENERGY REQUIREMENT AND HIGHEST OPERATING PRESSURE}

Minimum Energy of Desalination. Specific energy of desalination, $E$, is defined as the energy needed to produce a unit volume of product water. The theoretical minimum specific energy, $E_{\min }$, is equivalent to the Gibbs free energy of separation, $\Delta G_{\text {sep }}$, and can be simplified to ${ }^{26,36}$

$$
E_{\mathrm{min}}=\Delta G_{\mathrm{sep}} \approx \frac{\pi_{\mathrm{f}}}{Y} \ln \frac{1}{1-Y}
$$

where $\pi_{\mathrm{f}}$ is the feed stream osmotic pressure and $Y$ is the recovery rate. ${ }^{26,36}$ Previous studies showed that carrying out a hypothetical thermodynamically reversible RO desalination process with perfectly selective membranes (i.e., $100 \%$ salt rejection and, thus, permeate TDS concentration of zero) yields exactly the same expression as eq $1 .^{26,36}$ Because no entropy is produced in a thermodynamically reversible process, the energy consumed is, thus, the theoretical minimum.

Practical Specific Energy of Facility-Scale RO Desalination. Actual facility-scale desalination processes are not thermodynamically reversible and, hence, the practical energy requirement will be greater than $E_{\min }{ }^{26,34,36}$ Here analytical expressions for the practical specific energies of COMRO, DPRO, and CF/OARO are presented and briefly discussed, while details on the derivation of $E$ are in the Supporting Information (SI). The energy consumption in a stage is the product of the applied hydraulic pressure, $\Delta P$, and permeate volume, $\Delta V$. For an $N$-stage process, $E$ is the sum of the energy requirement along all the stages, divided by the overall permeate volume, $V_{\mathrm{p}}$. This is algebraically described as $E=$ 
$\sum(\Delta P \Delta V) / V_{\mathrm{p}}$. The practical specific energies to desalinate a saline feed of osmotic pressure $\pi_{\mathrm{f}}$ to recovery $Y$ with COMRO, DPRO, and CF/OARO can be expressed by eqs 2,3 , and 4, respectively:

$$
\begin{aligned}
& E_{\text {COMRO }}=k \pi_{\mathrm{f}}\left(1+N Y \sum_{i=1}^{N}\{[N+Y(N-i)](N-i Y)\}^{-1}\right. \\
& E_{\text {DPRO }}=k \pi_{\mathrm{f}}\left(\sum_{i=1}^{N}(N-i Y)^{-1}\right) \\
& E_{\mathrm{CF} / \text { OARO }}=\frac{k \pi_{\mathrm{f}}}{1-Y}
\end{aligned}
$$

where $k$, the operational overpressurization factor, signifies the portion of hydraulic pressure applied in excess of the maximum osmotic pressure difference within the stage to maintain adequate water flux for practical operation (refer to SI for further elaboration). $N$ denotes the number of $\mathrm{BCC}$ stages in COMRO and bilateral stages in CF/OARO (i.e., not counting the terminal RO stage); whereas in DPRO, $N$ includes all conventional RO stages in the series.

In practical desalination processes, operating parameters, such as $\Delta P$ and $\Delta V$ of each stage, can be varied while still yielding the same $Y$ overall. In this analysis, we consistently adopt a constant permeate increment approach for the different technologies, where in COMRO and DPRO the permeate volume is equally distributed among the BCC stages and conventional RO stages, respectively. Note that the working principles of CF/OARO dictate that the same volume of water permeates across each stage. Hence, the evaluation criteria are maintained the same among the technologies compared. An alternative scheme for operating the stages to yield the same $Y$, but by having a constant increment of $\Delta P$ between stages instead, is assessed in the SI.

Lower Operating Pressures are Needed for COMRO Compared to Conventional RO. The operating hydraulic pressures for the stages of cascading osmotically mediated RO (COMRO), direct pass RO (DPRO), and counter flow/ osmotically assisted RO (CF/OARO) are calculated using eqs S9, S11, and S13 in the SI, respectively. Figure 4 (symbols, right vertical axis) shows the highest applied hydraulic pressure, $\Delta P_{\max }$, required in single-stage $\mathrm{RO}, \mathrm{COMRO}, \mathrm{DPRO}$, and CF/ OARO for two scenarios: (A) desalinating hypersaline feed at 70000 ppm TDS to $50 \%$ recovery and (B) high recovery seawater desalination with $Y=70 \%$ (feed salinity, $c_{\mathrm{f}}$, of 35000 ppm TDS). Consideration for $\Delta P_{\max }$ in COMRO and CF/ OARO includes bilateral stages as well as terminal RO stage. Highest operating pressure for DPRO occurs in the final stage of the series. Configurations with one to four stages, not counting the terminal RO stage, are evaluated for COMRO and CF/OARO, while DPRO is assessed for $N=2-5(N=1$ is equivalent to single-stage $\mathrm{RO}$ ). That is, for the same total number of stages, $N$ of DPRO is one greater than $N$ of COMRO and CF/OARO. Horizontal gray dotted lines signify $85 \mathrm{bar}$, the approximate upper $\Delta P$ operating range of typical membrane modules. The operating hydraulic pressures and specific energy consumption for each stage are detailed in Tables S1-S6 of the SI.
Desalinating hypersaline feed at $70000 \mathrm{ppm}$ TDS to $50 \%$ recovery requires an applied hydraulic pressure of $137 \mathrm{bar}$ in single-stage RO (Figure 4A), substantially exceeding the typical operating $\Delta P$ of conventional RO membrane modules. DPRO also needs the equally high $\Delta P_{\max }$ of 137 bar, regardless of the number of stages. This is because the exiting retentate stream of the final stage in DPRO has identical salinity and, thus, osmotic pressure as the effluent brine of conventional RO (Figure 1B). The technical limitations encountered at the very high pressurizations, as discussed earlier, hence exclude single-stage $\mathrm{RO}$ and multistage DPRO from treating very high salinity feeds.

In contrast, $\Delta P$ required in COMRO to desalinate hypersaline feed are well within the 85 bar operating pressure limit. For all configurations of $N=1-4$, the most pressurized stage is at 68.3 bar (Figure 4A). Likewise, for CF/OARO, $\Delta P_{\max }$ is also 68.3 bar for the configurations evaluated. Both COMRO and CF/OARO are able to operate at lower $\Delta P_{\max }$ than single-stage $\mathrm{RO}$ and $\mathrm{DPRO}$, because the $\mathrm{BCC} /$ bilateral stages utilize saline stream on the dilute side to reduce the osmotic pressure difference that needs to be overcome (Figures 2 and 3). Further, the salinities handled by the terminal RO stage is significantly lessened compared to single-stage RO (and the last stage of DPRO) due to stepwise dilution in the preceding BCC/bilateral stages, thus markedly decreasing $\Delta P$. Therefore, COMRO and CF/OARO innovatively avoid the high membrane pressurizations, sidestepping the technical barrier to enable the potential desalination of hypersaline feeds.

COMRO is More Energy Efficient for Desalinating Hypersaline Feed. The practical specific energy requirement, $E$, for single-stage RO, COMRO, DPRO, and CF/OARO are compared in Figure 4 (columns, left vertical axis). The same scenarios of (A) hypersaline desalination and (B) high recovery seawater desalination, and stage configurations for the different technologies are assessed using eqs 2, 3, and 4. Pump and ERD inefficiencies, parasitic pressure drops along membrane modules, pre- and post-treatment costs, and intake and outfall power consumption are not factored into the energy requirement calculations. The theoretical minimum energy of desalination, $E_{\min }$, calculated using eq 1 , is also presented.

If single-stage RO is used to desalinate hypersaline feed of $70000 \mathrm{ppm}$ TDS to $50 \%$ recovery, $3.79 \mathrm{kWh}$ will be needed to produce $1 \mathrm{~m}^{3}$ of freshwater (Figure 4A, using eq 3 with $N=1$ ). The energy requirement is significantly greater than $E_{\min }$ of 2.29 $\mathrm{kWh} / \mathrm{m}^{3}$. To achieve the same desalination, COMRO requires $3.79,3.35,3.22$, and $3.16 \mathrm{kWh} / \mathrm{m}^{3}$ with one, two, three, and four BCC stages, respectively (eq 2). Therefore, utilizing COMRO with $N=2$ attains $11.7 \%$ reduction in $E$, while higher energy savings of up to $16.7 \%$ are achieved by increasing the number of bilateral countercurrent stages to three and four (Figure 4A). In contrast, CF/OARO consumes the same 3.79 $\mathrm{kWh} / \mathrm{m}^{3}$ regardless of number of stages (eq 4 is independent of $N)$ and has exactly identical energy efficiency as single-stage RO (substituting $N=1$ into eq 3 yields eq 4 ). Provided the $\Delta P$ technical impediments described earlier are addressed, DPRO offers the largest energy saving in hypersaline desalination compared to conventional single-stage RO, with $16.7 \%$ reduction in $E$ for two stages and further energy savings of up to $25.4 \%$ as $N$ increases to five (Figure $4 \mathrm{~A}$, using eq 3 ). The energy efficiencies of the different technologies are DPRO > COMRO > CF/OARO = single-stage RO.

The enhanced energy efficiency of COMRO and DPRO in desalinating high-salinity feeds, relative to single-stage RO and $\mathrm{CF} / \mathrm{OARO}$, can be graphically visualized using representative 
pressure-volume plots, as depicted in Figure 5. For parity in comparison between the desalination technologies, COMRO

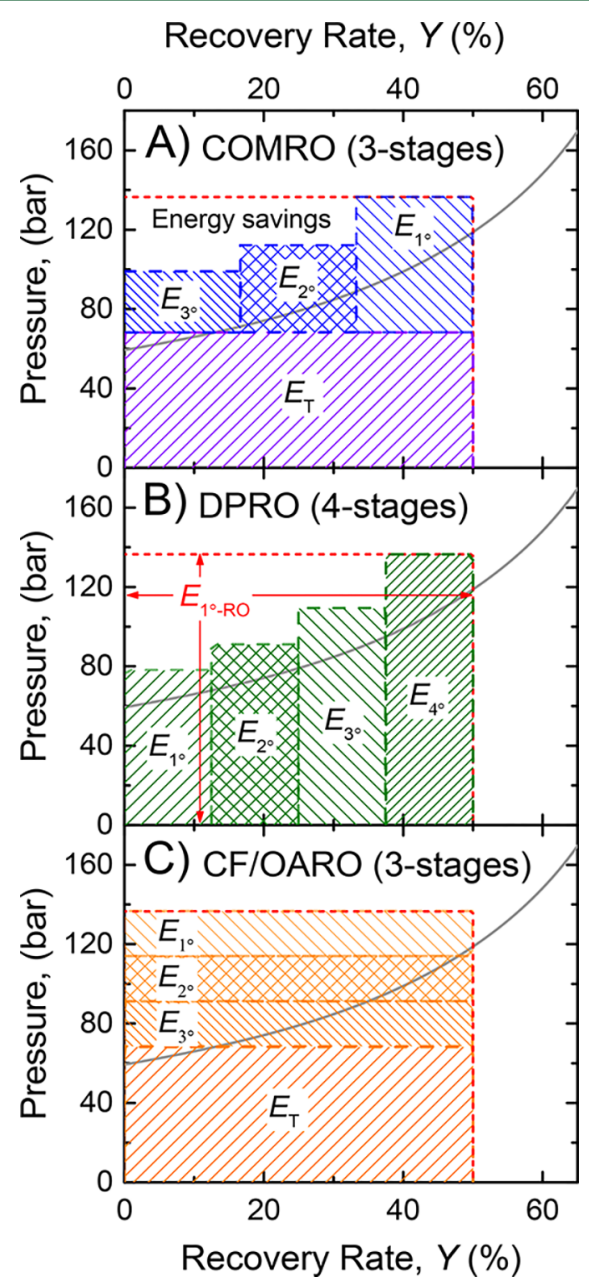

Figure 5. Energy requirement to desalinate hypersaline feed of $c_{\mathrm{f}}=$ $70000 \mathrm{ppm}$ TDS to $50 \%$ recovery for (A) 3-stage COMRO, (B) 4stage DPRO, and (C) 3-stage CF/OARO represented on hydraulic pressure-recovery rate plots, that is, equivalently, pressure-volume plots. The solid gray lines denote osmotic pressure of the retentate brine as a function of recovery. The area of the patterned regions represents the energy required in each stage, while the area under the dotted red lines indicates the energy consumption of conventional single-stage $\mathrm{RO}, E_{1^{\circ} \text {-RO }}$. The operational overpressurization factor, $k$, is 1.15 .

and CF/OARO are analyzed for $N=3$, i.e., three BCC/bilateral stages plus terminal RO stage for a total of four stages, the same number of stages considered in DPRO configuration. The area of the patterned regions represents the energy required in each stage and thus the sum of the areas, $\sum(\Delta P \Delta V)$, is proportional to $E$. The solid gray lines denote the osmotic pressure of the retentate stream as a function of recovery, that is, $\pi_{f} /(1-Y)$.

To desalinate hypersaline feed of $70000 \mathrm{ppm}$ TDS to $50 \%$ recovery in single-stage $\mathrm{RO}, \Delta P$ is 137 bar (horizontal red dashed lines in Figures 5A-C. Multiplying the pressure by the permeate volume, or equivalently the recovery, yields the energy requirement and is represented in Figure 5 as the area under the red dashed lines, $E_{1^{\circ} \text {-RO }}$. In COMRO, because the stream salinity is sequentially lowered by the BCC stages (Figure 2), energy consumed in the terminal RO stage (region labeled $E_{\mathrm{T}}$ in Figure $5 \mathrm{~A}$ ) is significantly less than $E_{1^{\circ} \text {-RO. To }}$ drive water flux from the more saline retentate stream to the dilute side in the cascading stages, work is done by the applied hydraulic pressure. However, the normalized permeate volume in each countercurrent stage (width of patterned regions) is only a fraction of the overall recovery, and the $\Delta P$ required (height of areas) is osmotically mediated by the dilute stream. Therefore, adding the energy consumed in the three BCC stages $\left(E_{1^{\circ}}, E_{2^{\circ}}\right.$, and $E_{3^{\circ}}$ in Figure $\left.5 \mathrm{~A}\right)$ to $E_{\mathrm{T}}$ still yields a lower energy requirement than single-stage $\mathrm{RO}$. This energy savings is signified by the difference between the area under the red dashed line and the sum of the patterned areas.

DPRO employs a series of conventional RO stages with incrementally elevated hydraulic pressures for stepwise recovery of freshwater (Figure 1B). The width of the patterned regions in Figure 5B is representative of the permeate volume in each stage, while the stepped height indicates $\Delta P$ progressively ramping up to 137 bar. DPRO consumes less energy than COMRO because in the BCC stages of COMRO, essentially pure water that permeates across the membrane is combined with the saline stream on the dilute side. This mixing is counter to the separation objective of desalination, and the undesirable mixing entropy produced diminishes the net energy efficiency. In contrast, water permeation in the DPRO stages directly constitute the final yield and, thus, avoid the unproductive generation of entropy due to mixing. Therefore, for the same number of stages, DPRO is more energy efficient than COMRO (and also CF/OARO), irrespective of feed salinity and recovery rate. However, the realization of this benefit is contingent on the aforementioned high pressurization challenge being resolved.

Although CF/OARO utilizes bilateral stages to lessen the applied hydraulic pressure required, the recirculating loop design necessitates a permeate volume equivalent to the final product water volume to pass through all stages (Figure 3). This is graphically represented in Figure $5 \mathrm{C}$ by the patterned regions with low height (i.e., low hydraulic pressure required in each stage) but identically broad width equal to the process recovery rate of $50 \%$. Summing up $\triangle P \Delta V$ of the bilateral stages, $1-3^{\circ}$, and the terminal RO stage yields energy consumption exactly matching conventional single-stage RO. An examination of eq 4 reveals that the energy consumption of $\mathrm{CF} / \mathrm{OARO}$ is independent of the number of bilateral stages and, furthermore, substituting $N=1$ into eq 3 yields eq 4 , that is, $E_{\mathrm{CF} / \mathrm{OARO}}=E_{1^{\circ}-\mathrm{RO}}$. This somewhat counterintuitive finding on the low energy efficiency of CF/OARO can be explained by the mixing entropy produced in the loops when the permeated water mixes with the saline sweep streams. Because the bilateral stages are linked by closed recirculating loops, the entire permeate volume has to pass through each of the $N$ loops, thus cumulatively producing an extensive amount of entropy. As work done in each stage is the product of applied hydraulic pressure and permeate volume, potential energy efficiency gains by lower $\Delta P$ requirements are offset by the large $\Delta V=V_{\mathrm{p}}$ (Figure $5 \mathrm{C}$ ). Hence, while CF/OARO offers the advantage of reduced $\Delta P_{\max }$ the process is disadvantaged by excessive energy demand.

COMRO Enables Enhanced Recovery of Seawater Desalination with Moderate Pressures and Substantial Energy Savings. The specific energy requirement and $\Delta P_{\max }$ to desalinate seawater to the high recovery rate of $70 \%$ are presented in Figure 4B. The same assessment method as scenario (A) of hypersaline desalination is employed. For conventional single-stage RO and DPRO, $\Delta P_{\max }$ is 114 bar, 
again surpassing the 85 bar pressure limit (horizontal gray dotted line). On the other hand, COMRO is able to achieve high recovery seawater desalination while operating with moderate pressures below 79.6 bar (symbols in Figure 4B). The operating pressure requirement among the technologies is lowest for CF/OARO, with $\Delta P_{\max }$ ranging between 34.1 and 79.6 bar, depending on $N$.

The trends in $E$ for high recovery seawater desalination are similar to the earlier scenario of hypersaline feed. Irrespective of number of stages, $\mathrm{CF} / \mathrm{OARO}$ has the same $E$ as single-stage $\mathrm{RO}$ of $3.16 \mathrm{kWh} / \mathrm{m}^{3}, 2.25$ times the thermodynamic limit of $1.42 \mathrm{kWh} / \mathrm{m}^{3}$. Hypothetically setting aside the anticipated technical problems at high pressurizations, DPRO attains the greatest energy efficiencies (26.9-40.7\% decrease in $E$, relative to single-stage RO). While not as efficient as DPRO, substantial gains in energy savings are still achieved using COMRO: energy reductions are enhanced from 23.0 to $32.9 \%$ as $N$ increases from one to four (columns in Figure 4B). Thus, among the desalination methods assessed in this study, COMRO is the only technology that simultaneously offers the benefits of improved energy efficiency and moderate hydraulic pressure requirements. Furthermore, the configuration of COMRO in this analysis is not optimized for either largest energy saving or minimum $\Delta P_{\max }$. In actual implementation, the flexibility of COMRO enables the process to be extensively customized (e.g., number of cascading stages, water permeation in each stage, and applied pressure in BCC and terminal stages) to meet specific operational and performance objectives.

\section{CAPITAL COST AND PRACTICAL CONSIDERATIONS}

Impact of Capital Expense on Levelized Cost of Water. The levelized cost of water (LCOW) is defined as the constant price to produce a unit volume of freshwater to breakeven over the lifetime of the desalination facility. In addition to energy consumption, LCOW also depends on the initial capital expenditure, expenses for chemicals, and other operational, financial, and indirect costs. ${ }^{42}$ Hence, LCOW is a compendious indicator that describes the economic feasibility of desalination. Capital cost, which includes system components such as membrane modules, high-pressure pumps, and ERDs, accounts for approximately $37-54 \%$ of LCOW in conventional RO desalination plants and is, thus, a critical factor for process viability. ${ }^{24,43,44}$

Increasing the number of stages reduces the specific energy demand for COMRO and DPRO, but capital expense will also unavoidably rise and, in turn, negatively impact LCOW. First, total membrane area required increases with more stages, regardless of the technology. However, the different technologies require different membrane transport properties. For the $\mathrm{BCC}$ stages in COMRO, since the main objective is to dilute the feed stream rather than generate high-quality product water, some leakage of salt across to the feed side is tolerable. Therefore, more water permeable membranes with comparatively lower salt selectivity can be employed to significantly lower the membrane area requirement. On the other hand, all stages in DPRO directly yield product water and are, therefore, constrained to more selective but less permeable membranes to maintain output water quality. Second, a greater number of stages also necessitates more auxiliary engineering components. Each added stage in DPRO and COMRO needs an additional booster pump to adjust the applied hydraulic pressure, while the capital equipment requirement for another bilateral stage in
$\mathrm{CF} / \mathrm{OARO}$ is even higher, with a pair of high-pressure pump and ERD needed, respectively, to pressurize and depressurize the sweep stream (Figures 1B, 2, and 3). Therefore, the least LCOW is achieved by optimizing the number of stages to balance the trade-off between operating energy cost and initial capital expense.

The steady-state operation of CF/OARO requires a constant volumetric permeation rate to be maintained throughout all stages, a process objective that will likely pose challenges in execution. Further, because the membranes are not perfectly selective (i.e., salt rejection $<100 \%$ ), salts from the feed stream that leak across the bilateral stages will inevitably accumulate in the closed recirculating loops of CF/OARO. The buildup of foreign salts and compounds in the working solutions will upset the delicate osmotic pressure balance needed for proper process function. The sweep stream is also anticipated to require periodic bleed and replenishment with new working solutions. These CF/OARO operating and maintenance cost will unfavorably affect LCOW.

Practical Implementation of COMRO to Extend Desalination Capabilities. Rapid implementation of COMRO in practice can be realized by appending the technology to existing desalination facilities for enhancement of overall recovery yields and, thus, augment water production capacity. This is illustrated in Figure 6A, where a 1-stage COMRO is added-on to conventional seawater RO (gray and blue shaded boxes, respectively). Note that other desalination methods, such as thermal distillation, can be integrated with COMRO as well. Flow rate, salinity, and osmotic pressure of the streams are indicated in the schematic. Exiting brine from the conventional $\mathrm{RO}$, after depressurization, is directed to the COMRO bilateral countercurrent stage for dilution and then to the terminal RO stage to produce more permeate. Freshwater production of the integrated process is further increased by $50 \%$ and, thus, net $Y$ is improved to $75 \%$.

In the COMRO configuration depicted in Figure $6 \mathrm{~A}, \Delta P$ applied in the BCC and terminal RO stages are both 68.3 bar, within the $\approx 85$ bar constraint. The identical pressurization further eliminates the need for an ERD for the retentate stream exiting the terminal RO stage (and before entering the BCC stage), thus trimming capital expenditure. Specific energy requirements of the stages are detailed in Figure $6 \mathrm{~B}$. The conventional single-stage RO requires $E$ of $1.90 \mathrm{kWh} / \mathrm{m}^{3}$ to attain $50 \%$ recovery yield (gray shaded area). Deploying the COMRO extension to increase overall $Y$ by $1.5 \times$ raises the specific energy demand by only $1.33 \times$ to $2.53 \mathrm{kWh} / \mathrm{m}^{3}$ (total shaded area). In comparison, to achieve $75 \%$ recovery in one conventional RO stage would consume $2 \times$ more energy $(E=$ $3.79 \mathrm{kWh} / \mathrm{m}^{3}$, area under dotted red lines) and require the prohibitively high $\Delta P$ of 136.6 bar.

Additionally, COMRO affords great flexibility in configuration of the stages and can be designed to treat challenging brines significantly more saline than the 70000 ppm TDS scenario examined earlier, without exceeding the technical constrain in $\Delta P$. For example, 2-stage COMRO can be utilized to concentrate hypersaline streams at $150000 \mathrm{ppm}$ TDS by $25 \%$ (i.e., equivalent to $Y$ of $20 \%$, refer to Figure S6 and discussion in SI). Hence, COMRO shows great promise to extend the capabilities of membrane-based technologies beyond seawater desalination. 

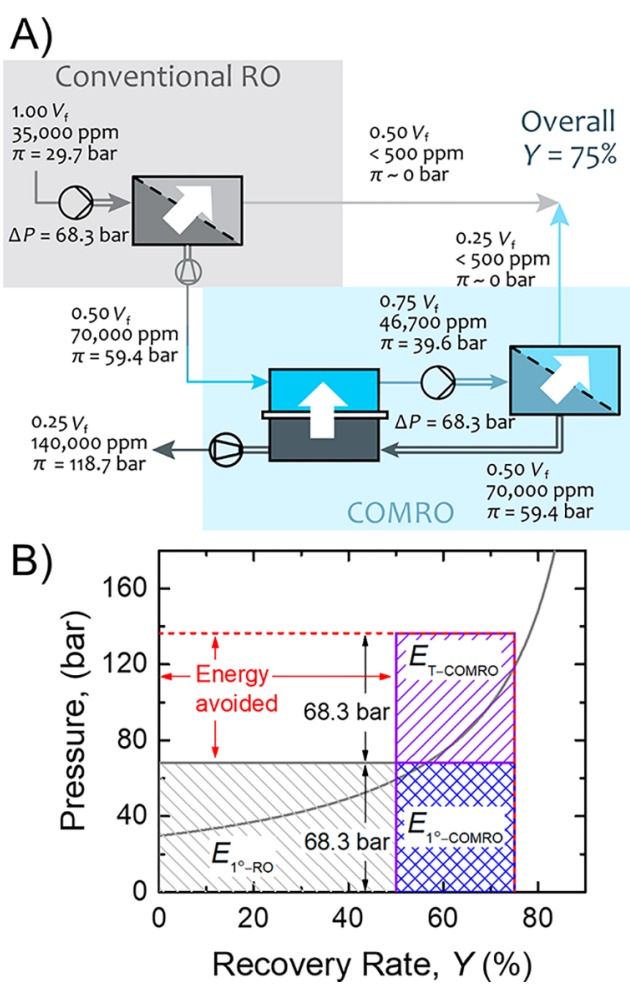

Figure 6. COMRO integrated with conventional single-stage RO to desalinate seawater feed of $c_{\mathrm{f}}=35000 \mathrm{ppm}$ TDS to overall recovery, $Y$, of $75 \%$. (A) Schematic of a 1-stage COMRO (blue shaded area) integrated with conventional RO (gray shaded area). Volumetric flow rate (relative to input feed of $1.00 V_{\mathrm{f}}$ ), TDS concentration, and osmotic pressure of the streams are indicated. (B) Specific energy requirement of the integrated process. The solid gray line denotes osmotic pressure of the retentate brine as a function of recovery. The area of the shaded regions represents the energy required in each stage. Difference between the area under the dotted red line and sum of shaded regions indicates the energy cost avoided by not carrying out the desalination in a conventional single-stage RO. Osmotic pressures are determined using the van't Hoff equation with temperature at 298 $\mathrm{K}$ and the operational overpressurization factor, $k$, is 1.15 .

\section{IMPLICATIONS}

The proposed cascading osmotically mediated reverse osmosis technology offers the dual advantages of high energy efficiency desalination with only moderate hydraulic pressure requirements. Presently, feed streams with salinity greater than seawater are beyond the reach of membrane-based processes and have to be treated by energy-intensive thermal processes. $^{1,11}$ COMRO can innovatively sidestep hydraulic pressure limitations imposed by the membrane module and extend the capabilities of membrane-based techniques to desalinate hypersaline brines and enhance recovery in seawater desalination. The technology has potential to treat ultrahighsalinity streams of emerging importance, such as desalinating produced water from oil and gas operations and dewatering brine from minimum/zero liquid discharge operations.

In the bilateral stages of COMRO, both the feed and permeate sides are saline, whereas conventional RO only has saline solution on the feed side. Due to the radically different working principles, current commercial $\mathrm{RO}$ membranes are likely unsuitable for the BCC stages. Mass transfer in the bilateral stages will be more similar to osmotically driven processes of forward osmosis and pressure retarded osmosis. ${ }^{45-47}$ Furthermore, since the aim of the BCC stages is to dilute the feed stream and not yield product water, the membrane active layer can be optimally tuned to the right balance of high water permeability while retaining adequate solute rejection. Therefore, development of membranes with specifically tailored structural and transport properties will be pivotal to enabling COMRO. Fouling is a pervasive problem plaguing all membrane processes. ${ }^{33}$ Membrane fouling, especially mineral scaling, ${ }^{48}$ will inescapably be a key issue when treating high-salinity streams in COMRO as well.

\section{ASSOCIATED CONTENT}

Supporting Information

The Supporting Information is available free of charge on the ACS Publications website at DOI: 10.1021/acs.est.7b05774.

Derivation of analytical expressions for specific energy requirement and operating pressures of COMRO, DPRO, and CF/OARO; analysis results of operating hydraulic pressures and specific energy consumption; analysis of alternative operating scheme for COMRO and DPRO; impacts of stage operating schemes on the specific energy requirement; and application of COMRO to treat ultrahigh salinity brine; Tables S1-6; Figures S1-S6 (PDF)

\section{AUTHOR INFORMATION}

\section{Corresponding Author}

*Phone: +1 212 8542984; e-mail: n.y.yip@columbia.edu.

ORCID

Ngai Yin Yip: 0000-0002-1986-4189

\section{Notes}

The authors declare the following competing financial interest(s): A patent application has been filed based on the idea and findings reported in the manuscript.

\section{ACKNOWLEDGMENTS}

We acknowledge the helpful comments and suggestions of the anonymous reviewers in sharpening the discussions in the manuscript.

\section{REFERENCES}

(1) Shaffer, D. L.; Chavez, L. H. A.; Ben-Sasson, M.; Castrillon, S. R V.; Yip, N. Y.; Elimelech, M. Desalination and Reuse of High-Salinity Shale Gas Produced Water: Drivers, Technologies, and Future Directions. Environ. Sci. Technol. 2013, 47 (17), 9569-9583.

(2) Gregory, K. B.; Vidic, R. D.; Dzombak, D. A. Water management challenges associated with the production of shale gas by hydraulic fracturing. Elements 2011, 7 (3), 181-186.

(3) Vidic, R. D.; Brantley, S. L.; Vandenbossche, J. M.; Yoxtheimer, D.; Abad, J. D. Impact of shale gas development on regional water quality. Science 2013, 340 (6134), 1235009.

(4) Bond, R.; Veerapaneni, S. V. Zeroing in on ZLD technologies for inland desalination. J. AWWA 2008, 100 (9), 76.

(5) Tong, T.; Elimelech, M. The global rise of zero liquid discharge for wastewater management: drivers, technologies, and future directions. Environ. Sci. Technol. 2016, 50 (13), 6846-6855.

(6) Subramani, A.; Jacangelo, J. G. Treatment technologies for reverse osmosis concentrate volume minimization: a review. Sep. Purif. Technol. 2014, 122, 472-489.

(7) Clark, C.; Veil, J. Produced Water Volumes and Management Practices in the United States; Argonne National Laboratory (ANL): 2009.

(8) Benko, K. L.; Drewes, J. E. Produced water in the Western United States: geographical distribution, occurrence, and composition. Environ. Eng. Sci. 2008, 25 (2), 239-246. 
(9) Keranen, K. M.; Weingarten, M.; Abers, G. A.; Bekins, B. A.; Ge, S. Sharp increase in central Oklahoma seismicity since 2008 induced by massive wastewater injection. Science 2014, 345 (6195), 448-451.

(10) Heijman, S.; Guo, H.; Li, S.; Van Dijk, J.; Wessels, L. Zero liquid discharge: heading for $99 \%$ recovery in nanofiltration and reverse osmosis. Desalination 2009, 236 (1-3), 357-362.

(11) Ahmadun, F. R.; Pendashteh, A.; Abdullah, L. C.; Biak, D. R. A.; Madaeni, S. S.; Abidin, Z. Z. Review of technologies for oil and gas produced water treatment. J. Hazard. Mater. 2009, 170 (2-3), 530551.

(12) Thiel, G. P.; Tow, E. W.; Banchik, L. D.; Chung, H. W.; Lienhard, J. H. Energy consumption in desalinating produced water from shale oil and gas extraction. Desalination 2015, 366, 94-112.

(13) Carati, A.; Marino, M.; Brogioli, D. Thermodynamic study of a distiller-electrochemical cell system for energy production from low temperature heat sources. Energy 2015, 93, 984-993.

(14) Brogioli, D.; La Mantia, F.; Yip, N. Y. Thermodynamic analysis and energy efficiency of thermal desalination processes. Desalination 2018, 428, 29-39.

(15) Coday, B. D.; Xu, P.; Beaudry, E. G.; Herron, J.; Lampi, K.; Hancock, N. T.; Cath, T. Y. The sweet spot of forward osmosis: Treatment of produced water, drilling wastewater, and other complex and difficult liquid streams. Desalination 2014, 333 (1), 23-35.

(16) Pendergast, M. M.; Nowosielski-Slepowron, M. S.; Tracy, J. Going big with forward osmosis. Desalin. Water Treat. 2016, 57 (55), 26529-26538.

(17) Alkhudhiri, A.; Darwish, N.; Hilal, N. Produced water treatment: Application of Air Gap Membrane Distillation. Desalination 2013, 309, $46-51$.

(18) Drioli, E.; Ali, A.; Macedonio, F. Membrane distillation: Recent developments and perspectives. Desalination 2015, 356, 56-84.

(19) Tsai, J. H.; Macedonio, F.; Drioli, E.; Giorno, L.; Chou, C. Y.; Hu, F. C.; Li, C. L.; Chuang, C. J.; Tung, K. L. Membrane-based zero liquid discharge: Myth or reality? J. Taiwan Inst. Chem. Eng. 2017, 80, 192-202.

(20) Mistry, K. H.; McGovern, R. K.; Thiel, G. P.; Summers, E. K.; Zubair, S. M.; Lienhard, J. H. Entropy generation analysis of desalination technologies. Entropy 2011, 13 (10), 1829-1864.

(21) Spiegler, K.; El-Sayed, Y. The energetics of desalination processes. Desalination 2001, 134 (1), 109-128.

(22) Lin, S.; Yip, N. Y.; Elimelech, M. Direct contact membrane distillation with heat recovery: Thermodynamic insights from module scale modeling. J. Membr. Sci. 2014, 453, 498-515.

(23) Fritzmann, C.; Lowenberg, J.; Wintgens, T.; Melin, T. State-ofthe-art of reverse osmosis desalination. Desalination 2007, 216 (1-3), $1-76$.

(24) Greenlee, L. F.; Lawler, D. F.; Freeman, B. D.; Marrot, B.; Moulin, P. Reverse osmosis desalination: Water sources, technology, and today's challenges. Water Res. 2009, 43 (9), 2317-2348.

(25) Global Water Intelligence: Monthly Analysis and Data on the Global Water Market; Media Analytics: Oxford, U.K., 2000; p v.

(26) Elimelech, M.; Phillip, W. A. The Future of Seawater Desalination: Energy, Technology, and the Environment. Science 2011, 333 (6043), 712-717.

(27) Rautenbach, R.; Linn, T.; Eilers, L. Treatment of severely contaminated waste water by a combination of RO, high-pressure RO and NF - potential and limits of the process. J. Membr. Sci. 2000, 174 (2), 231-241.

(28) Coday, B. D.; Heil, D. M.; Xu, P.; Cath, T. Y. Effects of Transmembrane Hydraulic Pressure on Performance of Forward Osmosis Membranes. Environ. Sci. Technol. 2013, 47 (5), 2386-2393.

(29) Hickenbottom, K. L.; Vanneste, J.; Elimelech, M.; Cath, T. Y. Assessing the current state of commercially available membranes and spacers for energy production with pressure retarded osmosis. Desalination 2016, 389, 108-118.

(30) Straub, A. P.; Osuji, C. O.; Cath, T. Y.; Elimelech, M. Selectivity and Mass Transfer Limitations in Pressure-Retarded Osmosis at High Concentrations and Increased Operating Pressures. Environ. Sci. Technol. 2015, 49 (20), 12551-12559.
(31) Warsinger, D. M.; Tow, E. W.; Nayar, K. G.; Maswadeh, L. A.; Lienhard, J. H. Energy efficiency of batch and semi-batch (CCRO) reverse osmosis desalination. Water Res. 2016, 106, 272-282.

(32) Lin, S. H.; Elimelech, M. Staged reverse osmosis operation: Configurations, energy efficiency, and application potential. Desalination 2015, 366, 9-14.

(33) Baker, R. W. Membrane Technology and Applications, 3rd ed.; John Wiley \& Sons: Hoboken, 2012.

(34) Mulder, M. Basic Principles of Membrane Technology, 2nd ed.; Kluwer Academic: Dordrecht, 1996; p 564 p.

(35) Song, L. F.; Hu, J. Y.; Ong, S. L.; Ng, W. J.; Elimelech, M.; Wilf, M. Emergence of thermodynamic restriction and its implications for full-scale reverse osmosis processes. Desalination 2003, 155 (3), 213228.

(36) Liu, C.; Rainwater, K.; Song, L. F. Energy analysis and efficiency assessment of reverse osmosis desalination process. Desalination 2011, $276(1-3), 352-358$.

(37) Busch, M.; Mickols, W. E. Reducing energy consumption in seawater desalination. Desalination 2004, 165 (1-3), 299-312.

(38) Zhu, A. Z.; Christofides, P. D.; Cohen, Y. Effect of Thermodynamic Restriction on Energy Cost Optimization of RO Membrane Water Desalination. Ind. Eng. Chem. Res. 2009, 48 (13), $6010-6021$.

(39) RO Technology Concentrates Brine. Water. Desalin. Rep. 2017, 53, (17), 3.

(40) Choong, L. T.; Govindan, P. N.; St. John, M. G.; Lam, S.; Andrews, J.-R.; Chehayeb, K. M. Osmotic desalination methods and associated systems. WO/2017/019944, 2017.

(41) Bartholomew, T. V.; Mey, L.; Arena, J. T.; Siefert, N. S.; Mauter, M. S. Osmotically assisted reverse osmosis for high salinity brine treatment. Desalination 2017, 421 (Supplement C), 3-11.

(42) Caldera, U.; Bogdanov, D.; Breyer, C. Local cost of seawater RO desalination based on solar PV and wind energy: A global estimate. Desalination 2016, 385, 207-216.

(43) Younos, T. The economics of desalination. J. Contemp. Water Res. Educ. 2005, 132 (1), 39-45.

(44) Miller, J. Review of Water Resources and Desalination Technologies; Sandia National Laboratories, 2003.

(45) Yip, N. Y.; Elimelech, M. Performance limiting effects in power generation from salinity gradients by pressure retarded osmosis. Environ. Sci. Technol. 2011, 45 (23), 10273-10282.

(46) Yip, N. Y.; Tiraferri, A.; Phillip, W. A.; Schiffman, J. D.; Elimelech, M. High performance thin-film composite forward osmosis membrane. Environ. Sci. Technol. 2010, 44 (10), 3812-3818.

(47) Kim, J.; Kim, D. I.; Hong, S. Analysis of an osmoticallyenhanced dewatering process for the treatment of highly saline (waste)waters. J. Membr. Sci. 2018, 548, 685-693.

(48) Antony, A.; Low, J. H.; Gray, S.; Childress, A. E.; Le-Clech, P.; Leslie, G. Scale formation and control in high pressure membrane water treatment systems: A review. J. Membr. Sci. 2011, 383 (1-2), 116. 